\title{
I Fórum Nacional de Educação Farmacêutica: o farmacêutico que o Brasil necessita
}

\author{
First National Forum for Pharmaceutical Education: the pharmacist that Brazil needs
}

\begin{abstract}
A história do ensino farmacêutico no Brasil (com início datado de 1832) pode ser contada com base nas tentativas de alteração e uniformização curriculares. Mais expressivamente, influem sobre a situação atual os currículos mínimos de 1962 (parecer CFE 268) e de 1969, que regularam a graduação em farmácia até 2002. Na década de 1980 o papel do farmacêutico na sociedade passou a ser tema de discussão na categoria, acompanhado necessariamente da discussão sobre a educação farmacêutica.

A publicação das Diretrizes Curriculares Nacionais de 2002 - DCN (resolução CNE/CES 02/2002) foi um importante marco histórico para a profissão ao determinar que a formação do farmacêutico deve ter como foco a preparação para atuar no Sistema Único de Saúde - SUS, articulado ao contexto social, participando e contribuindo para o desenvolvimento da sociedade. Após a publicação das DCN, as dificuldades geradas na sua interpretação e aplicação direcionaram as discussões na área da educação farmacêutica, que ficaram concentradas nas formas de implementar as novas diretrizes.
\end{abstract}

O Departamento de Assistência Farmacêutica e Insumos Estratégicos (DAF/SCTIE/MS) tem como um de seus eixos de atuação a qualificação da assistência farmacêutica no âmbito do SUS. Qualificar um serviço de saúde compreende, necessariamente, contar com profissionais adequadamente formados e preparados para gerenciar o serviço e as pessoas, inovar, integrar e cuidar, com base em critérios científicos e éticos para a consolidação dos princípios do SUS. Portanto, a questão da qualificação profissional, e em especial do farmacêutico, é fundamental para a estruturação de serviços de Assistência Farmacêutica - AF qualificados em todos os níveis de gestão.

O I Fórum Nacional de Educação Farmacêutica (realizado dias 13 e 14 de dezembro de 2007 em Brasília) foi uma iniciativa do DAF/SCTIE/MS em parceria com a Associação Brasileira de Ensino Farmacêutico (ABENFAR). Seu objetivo foi debater, com entidades representativas da classe farmacêutica, estudantes, professores de farmácia e representantes dos serviços de saúde, as prioridades para a formação do profissional farmacêutico para atender as necessidades do país e do SUS.
O tema central para o Fórum, "O farmacêutico que o Brasil necessita", teve por objetivo instigar a categoria para a construção de propostas para a educação diretamente relacionadas com a realidade social do país e as demandas da sociedade, especialmente do SUS, para o farmacêutico. A programação do I Fórum privilegiou a participação ativa dos presentes, por meio de "espaços de diálogo" formados por quatro grupos de discussão.

A categorização dos debates e propostas construídas durante 0 evento estão, resumidamente, apresentadas a seguir:

- Percepção da situação atual: os participantes relataram a percepção de que a educação farmacêutica ainda é desenvolvida de forma desarticulada da realidade social, pouco comprometida com a resolução dos problemas nacionais de saúde. A formação é focada na produção de medicamentos, técnicas e exames, enquanto a saúde da população tem pouco espaço no processo educativo. Pouca interação academia-serviços e escassas atividades interdisciplinares também foram relatadas.

- Imagem-objetivo - a educação para o farmacêutico que o Brasil necessita: o farmacêutico como profissional de saúde, preparado para o SUS, capaz de intervir científica e criticamente sobre os problemas de saúde e sobre o sistema de saúde, com competência para promover a integralidade da atenção à saúde, de forma ética e interdisciplinar, e valorizado pela sociedade, constitui o 
objetivo a ser alcançado por meio da educação, segundo os participantes do evento.

a) Propostas gerais: rediscutir a proposta do currículo generalista (regido pela resolução 02/2002), buscando uma identidade central na formação farmacêutica baseada na assistência farmacêutica (desde a produção até a dispensação), de forma integralmente articulada aos princípios do SUS; estimular a discussão política nos cursos e ampliar a participação do Ministério da Educação no processo de mudança da formação farmacêutica.

b) Sobre a oferta de pósgraduação: estimular a criação e direcionamento da pós-graduação para as necessidades do SUS em AF, gerando condições para a inclusão de profissionais dos serviços de saúde nos programas; determinar a responsabilidade de farmacêuticos, serviços de saúde e programas de pósgraduação na geração e aplicação de pesquisas, conhecimentos e práticas direcionados para contribuir com a melhoria dos serviços de AF e da qualidade de vida da população; fomentar e fortalecer os programas de residência multiprofissional.

c) Sobre o fomento para a pesquisa: promover a articulação entre os órgãos de fomento, regulação e o Ministério da Saúde para ampliação de fomento de pesquisa em estudos aplicados na AF e o cuidado em saúde; formalizar a subárea de conhecimento na área da farmácia no CNPq, que inclua a AF e temas relacionados; adequar a avaliação da pesquisa e as diretrizes editoriais para a área de AF.

d) Integração academia-serviços de saúde: promover atividades formativas de farmacêuticos, em nível de graduação e pós-graduação, em serviços de saúde em todos os níveis de atenção e todo o âmbito de atuação do farmacêutico, incluindo a gestão dos serviços e atenção básica; estabelecer diálogo com os gestores e formas regulamentadas de oferta de estágios no SUS.

e) Qualificação dos profissionais dos serviços: criar e implementar a educação continuada dos profissionais farmacêuticos atuantes nos serviços de saúde públicos e privados; desenvolver a formação de profissionais de nível técnico para o trabalho auxiliar ao farmacêutico nas unidades de saúde.

f) Avaliação dos cursos de graduação: promover a definição da carga horária mínima; definir a necessidade de submissão das propostas de abertura de novos cursos de farmácia ao controle social e/ou a outras formas de regulação; inserir formas de avaliação das mudanças no modelo de formação do farmacêutico para o atendimento das necessidades do sistema de saúde no sistema de avaliação dos cursos de graduação do INEP.

Todas as propostas desenvolvidas pelos grupos de discussão foram apresentadas e comentadas por representantes de entidades diretamente relacionadas à educação farmacêutica: INEP, CAPES, SESU/MEC, DEGES/ MS, FENEFAR, ENEFAR, além do DAF/MS e ABENFAR.

A participação expressiva, motivada e entusiasmada dos professores, acadêmicos e profissionais farmacêuticos presentes no I Fórum Nacional de Educação Farmacêutica demonstrou o grande comprometimento da categoria na construção de um modelo de educação e de atuação farmacêutica que responda ativa e decisivamente às demandas sociais e ao desenvolvimento do país. Denota, ainda, o amadurecimento das reflexões em torno do farmacêutico que o Brasil necessita, definido nas propostas como um agente da saúde pública, tecnicamente preparado e politicamente consciente e ativo.

O relatório do evento pode ser acessado integralmente em < http://portal.saude.gov.br/portal/arquivos/pdf/ Relatorio_site_270308.pdf>

Silvana Nair Leite

Farmacêutica. Consultora técnica, Departamento de Assistência Farmacêutica e Insumos Estratégicos, Secretaria de Ciência, Tecnologia e Insumos Estratégicos, Ministério da Saúde. Departamento de Assistência Farmacêutica - CGAFB/DAF/SCTIE/ MS, Esplanada dos Ministérios, Bloco G, $8^{\circ}$ andar Brasília, DF - 70.058-900. silvana.leite@saude.gov.br

José Miguel do Nascimento Jr., DAF/SCTIE/MS.

Luiz Henrique Costa, Associação Brasileira de Ensino Farmacêutico.

Dirceu Aparecido Bras Barbano, DAF/SCTIE/MS. 